\title{
Randomized clinical trials in surgery: Why do we need them?
}

Charles M. Balch, MD, FACS

See related articles on pages $229,233,243,245,247$, and 249.
From the Departments of Surgery and Oncology, Johns Hopkins Medical Institutions, Baltimore, Md.

Received for publication Dec 28, 2005; accepted for publication March 3, 2006.

Correspondence: E-mail: balchch@jhmi. edu

J Thorac Cardiovasc Surg 2006;132:241-2

$0022-5223 / \$ 32.00$

Copyright $\odot 2006$ by The American Association for Thoracic Surgery

doi:10.1016/j.jtcvs.2006.03.039
$\mathrm{R}$ andomized clinical trials (RCTs) are a fundamental tool for surgical investigators to validate new therapies, but they are still used sparingly, and the results are reported in an inconsistent format. This conclusion is highlighted by the provocative article by Tiruvoipati and colleagues, ${ }^{1}$ who report in this issue on the use of the CONSORT statement, a checklist and flowchart used in reporting results of RCTs, in the cardiothoracic surgery literature. This important statement is endorsed by many medical journals but not by most surgical journals. I strongly agree with the authors' major conclusion that

It is therefore important that RCTs are reported in a high quality manner so the readers could have a clear view on why the study was conducted, how it was conducted and how it was analyzed. This would be helpful not only in the immediate appraisal of trials, but also in the long term, when performing further analyses such as in systematic reviews and meta-analyses. ${ }^{1}$

They also make the important point that a uniform reporting format about the design and methodology of a trial is vital, so that results of subsequent trials can be compared with those of previously published reports or so that trial results can be appropriately compared in a meta-analysis. If this is not done, there is a real risk that trial results would not be compared in a valid manner and, as a consequence, that a potentially effective therapy in surgical patients might be either discarded (because of false-negative results) or applied improperly (because of false-positive results).

The issue of randomized clinical trials is a conundrum for surgical investigators of all specialties. This is especially true in the surgical oncology field, where there are scores of new agents and new technologies that must be validated on the one hand, while the value to these new advances is beneficial to a smaller and smaller population of patients with cancer on the other hand, as we are able to distinguish smaller and smaller cancer subtypes based on genetic and molecular markers. We are now in an era of rapid and expensive research advances that will make it difficult to perform randomized trials requiring hundreds (or even thousands) of patients with narrow indications or eligibility for treatment. This means that we will need to examine alternative means, such as using surrogate or biologic end points other than multiyear survival rates. The capacity of completing major clinical trials in a timely manner within the referral practice of a single institution is diminishing rapidly. This means that surgical researchers will increasingly depend on multi-institutional (and even multinational) clinical trials to address the added or substitutive value of a new agent or new technology. In addition, the use of surrogate end points to validate new therapies and new statistical methodologies will allow for more timely, less expensive, but still accurate ways to assess rapidly advancing scientific and technologic knowledge as it applies to the surgical patient. Thus as we partition patients with cancer on the basis of more precise molecular markers and customize our treatment to the biology of smaller and smaller subpopulations of patients with cancer, will we still be able to perform randomized trials evaluating new combination treatments for efficacy and safety in a timely manner? Probably not.

This leads to the issue of the role of the surgeon in the conduct of cancer clinical trials. Certainly we as a surgical research community have used RCTs increasingly through the years, but this methodology is still underused, and even the results of such RCTs are not reported in a consistent enough format. This is especially true if we compare our use with the use of RCTs by our colleagues in medical oncology. 
At present, surgical oncologists do not have the training, the infrastructure, and the resources that compare with those of our counterparts in medical oncology practice, where RCTs have been imbedded into their clinical training and practice to a significant degree, both in academia and in private practice. I believe that a major effort must be made by surgical leaders to incorporate the capability of conducting clinical trials into our surgical training programs and for more surgical mentors to be role models for conducting clinical trials to a far greater extent than exists at present. Otherwise, we will not be in a position to test novel or innovative therapies and will default this responsibility to other specialties. This expanded role of the surgeon as a clinical trialist must span all aspects of the process: in the design phase (as a principle investigator or co-principle investigator), in the conduct of clinical trials, in the reporting of results, and in the knowledge of how (and when) to incorporate the results of clinical trials into new standards of care. If we continue to move in this direction, then our patients benefit, and the advances of new therapies will be incorporated more quickly and uniformly.

The authors of this article made a sage conclusion that ... the results of RCTs will have a significant impact on clinical decision making at all levels it is important to improve the quality of reporting. This may be achieved by the endorsement of the CONSORT statement by CT journals as well as increasing the awareness of CONSORT statement among the CT surgical researchers. ${ }^{1}$

This is so true, not only for patients undergoing cardiovascular surgery but also for those who are treated by all surgical subspecialties.

As a consequence of discussions with Dr Gene Blackstone during the review of this manuscript, I recommended to the Editorial Board of the Annals of Surgical Oncology that they adopt the CONSORT statement. They agreed, and we have added it to our "Instructions to Authors." Hopefully, other surgical journals will also adopt this important statement as well. I would also join the authors in encouraging surgical investigators of all specialties to use the CONSORT criteria when reporting their RCT results so that our studies will be more reproducible and comparable and so that our patients will maximally benefit.

\section{Reference}

1. Tiruvoipati R, Balasubramanian SP, Atturu G, Peek GJ, Elbourne D. Improving the quality of reporting randomized controlled trials in cardiothoracic surgery: the way forward. J Thorac Cardiovasc Surg. 2006;132:233-40 